Sharif University of Technology
Scientia Iranica
Transactions E: Industrial Engineering
SCIENTIA
I RAN I C A

\title{
Integrated production and distribution scheduling for perishable products
}

\author{
F. Marandi and S.H. Zegordi* \\ Department of Industrial Engineering, Tarbiat Modares University, Tehran, Iran.
}

Received 20 October 2015; received in revised form 18 April 2016; accepted 5 December 2016

\author{
KEYWORDS \\ Production and \\ distribution; \\ Permutation flow shop \\ scheduling; \\ Vehicle routing \\ problem; \\ Integration; \\ Mixed integer \\ programming; \\ Particle swarm \\ optimization.
}

\begin{abstract}
This study is concerned with how the quality of perishable products can be improved by shortening the time interval between production and distribution. Since special types of food, such as dairy products, decay fast, the Integration of Production and Distribution Scheduling (IPDS), is investigated. This article deals with a variation of IPDS that contains a short shelf life product; hence, there is no inventory of the product in the process. Once a specific amount of the product is produced, it must be transported with the least transportation time directly to various customer positions within its limited lifespans to minimize the delivery and tardy costs required to complete producing and distributing of the product to satisfy the demand of customers within the limited deadline. After developing a mixed-integer nonlinear programming model of the problem, because it is NP-hard, an Improved Particle Swarm Optimization (IPSO) is proposed. IPSO performance is compared with commercial optimization software for small-size and moderate-size problems. For large-size ones, it is compared with the genetic algorithm existing in the literature. Computational experiments show the efficiency and effectiveness of the proposed IPSO in terms of both the quality of the solution and the time of achieving the best solution.
\end{abstract}

(C) 2017 Sharif University of Technology. All rights reserved.

\section{Introduction}

Production and distribution operations are two important operational functions in a supply chain. To achieve optimal operational performance in a supply chain, it is significant to integrate these two functions and schedule them jointly in a coordinated manner. However, most of the proposed integrated and synchronized approaches focus on the tactical decision level of supply chains $[1,2]$. In recent years, integrated scheduling has attracted much interest among researchers. In contrast to classical scheduling, this type of scheduling problem involves not only the production part but also distribution. The objective of integrated scheduling is to obtain a simultaneous optimization

\footnotetext{
*. Corresponding author. Tel.: +982182883394

E-mail address: zegordi@modares.ac.ir (S.H. Zegordi)
}

of both parts. The obtained procedure will become a detailed schedule that provides an efficient solution for operation management. In this paper, a class of integrated scheduling problems is considered, which includes production and distribution.

In many applications involving make-to-order or time-sensitive (e.g., perishable) products, finished orders are often delivered to customers immediately or shortly after the production to restrict quality reduction. In such a supply chain, product quality is determined not only by the production processes but also through the coordination of the production and distribution decisions. In this situation, the delivery of the products must be done within a strictly limited time after their production. The non-inventory production and transportation problem is routine in many industries, in which a time-sensitive product cannot be in storage due to its short shelf life. The delivery of 
the product must be made within a tightly limited time after its production. Therefore, the production and distribution operations must be highly integrated. When the production plant has a limited production rate and the transportation time is not instantaneous, any inefficiency in the integrated schedule may either cause the product to expire before it reaches the customers or cause the delivery not to satisfy a customer's delivery deadline. Then, the production and distribution operations must be highly linked and integrated because any inefficiency in the integrated schedule causes a decrease in product quality, expiration before delivery, extra expenses, and lack of customer satisfaction. To ease this coordination, production sites are usually directly connected to customers by a fleet of vehicles [3]. Consequently, there is little or no finished product inventory in the supply chain such that production and outbound distribution are very closely linked, which must be scheduled jointly in order to achieve a desired on-time delivery performance at a minimum total cost. However, the analysis of practices in case studies show that, currently, the production and distribution operations are done separately, which cause operational and customer dissatisfaction [4]. Thus, this paper investigates integrating production and distribution decisions at a trade-off among customer satisfaction, quality of delivered products, and total costs. Research on integrated scheduling models of production and distribution is relatively new, but it is growing very rapidly.

This study was motivated by a practical scheduling problem encountered by a leading manufacturer of various industries, in which a limited number of vehicles were available, where the departure time after production in flowshop scheduling was not fixed and needed to be determined in order to minimize tardy and delivery costs to satisfy the customers' deadline. The differences and contributions of this paper compared with IPDS's literature can be summarized as follows:

1. A new problem is defined. The Integrated Production-Distribution Scheduling (IPDS) problem is considered, of which the first stage contains permutation flow shop scheduling and the second stage involves the distribution problem that requires designing vehicle routes for picking up finished goods and delivering them from the manufacturer to customers;

2. A new mixed integer nonlinear programming model is developed, which simultaneously considers both production and distribution scheduling in an integrated manner. The exact solution to the problem is provided by solving the model. This model is practical in large scales for real cases, such as the dairy product industry, which is motivated by a case study from a dairy manufacturer in Tehran;
3. An Improved Particle Swarm Optimization (IPSO) is proposed to deal with the problem. The improving operator, 1-exchanged and 2-opt, is added to prevent premature convergence;

4. New test problems are created, which can be used for future studies.

This paper studies the integrated production and distribution scheduling problem and extends features such as non-negligible transportation time and delivery consolidation. Comparison of this problem with previous works shows the combination of the product's limited lifespan, machine scheduling decisions, and the vehicle routing decisions as critical features that make it different, which leads to the possibility of expiration before it reaches a customer. The problem is complicated by limited transportation capacity, a customer's demand size and location, and the departure time that must be determined and are not fixed. On the other hand, these complications also make the resulting integrated scheduling problem challenging and interesting. The particular variation that is considered involves a single production plant with multiple production machines in flowshop scheduling, a fleet of delivery trucks, and a given set of customers at different locations over a geographic region.

The aim of this research is integrating scheduling in a mathematical model, which is developed to investigate the effect of integrated production scheduling and distribution decisions on the total costs. This is similar to various real-world environments such as food, dairy products, and chemical industries, which are highly perishable. Since this problem has an NPhard structure [5], a meta-heuristic method is used to solve it. An Improved Particle Swarm Optimization (IPSO) is proposed in this study that improves the operator, while 1-exchanged and 2-opt is added to prevent premature convergence.

The remainder of this paper is organized as follows. Section 2 presents results from previous works on integrated supply chain scheduling. In Section 3, the problem is described and the mathematical model of the problem is presented. In Section 4, an improved particle swarm optimization is proposed to solve the problem. In Section 5, the experimental results of the proposed IPSO and managerial implications are presented. At the end, Section 6 elaborates concluding remarks and some suggestions for future research.

\section{Literature review}

In this section, the literature that explicitly involves both production and transportation activities in integrated supply chain scheduling is presented. Integrating production and outbound delivery scheduling is very critical and common in the supply chain of time- 
sensitive products [6,7]. For example, Buer et al. [8] focus on the newspapers printing and distribution, and mail processing and distribution provided by Wang et al. [9], and industrial adhesive materials production and delivery provided by Devapriya et al. [10] Therefore, how to effectively integrate the production and delivery stages at the operational level so as to decrease the operational costs and improve customer service becomes very important for the success of a company. However, most of the existing models on the production-distribution scheduling problems only study strategic or tactical levels of decisions, and very few have addressed integrated decisions at the operational level. Chandra and Fisher [11] emphasize the need for studying these integrated scheduling issues at the operational level. They consider an integrated scheduling problem where a plant produces and stores the products until they are delivered to the customers by a fleet of trucks. They provide two solutions. The first solution solves the production scheduling and vehicle routing problems, separately, but the second one solves the problem in a coordinated manner. Their computational results show that the total operating cost decreases in the coordinated one. Chen and Vairaktarakis [12] and Pundoor and Chen [13] also show that there is significant benefit by using the optimally integrated production-distribution schedule compared to the schedule generated by a separate and sequential scheduling approach in the context of the models that are considered in their research. They emphasize that integration is a prior approach to improve the total performance in decreasing costs that is investigated in this paper.

Chen [6,14] reviews papers that deal with integrated production scheduling and outbound distribution. He focuses on two main areas of direct delivery (one destination) and vehicle routing (multi destinations). He also provides a survey of models and results in the area of integrated scheduling of production and distribution. He presents a unified model representation scheme, classifies existing models into several different classes, and, for each class of the models, gives an overview of the optimality properties, computational tractability, and solution algorithms for the various problems studied in the literature. In his survey on the relevant literature, he states that although there is a huge amount of research on integrated production and distribution models, the need for focusing on more studies in this realm is required. In his research, he notes a gap in the consideration of a due date and distribution by vehicle routing for practical situations. Lee and Chen [15] study the machine scheduling problems with explicit transportation considerations. They identify two types of transportation situations in their models. The first type involves transporting a semi-finished job from one machine to another for further processing. The second type involves transporting a finished job to the customer or warehouse. Both transportation capacity and transportation time are taken into account explicitly in their model. They classify the computational complexity of various scheduling problems by either proving their NP-hardness or providing polynomial algorithms. Chang and Lee [5] consider an extension of Lee and Chen's work where each job is assumed to occupy a different amount of storage space in the vehicle during delivery. They show the problem that jointly considers production and delivery with the consideration that each job may require a different amount of space during intractable transport and provide heuristics for some cases of the problem. Zhong et al. [16] study the similar problem as the one studied by [5] with the objective of minimizing the makespan. In the practical cases, focusing on the type of transportation, Hajiaghaei-Keshteli and Aminnayeri [17] present integrated production and rail transportation scheduling by emphasizing the application of rail distribution. Saidi-Mehrabad et al. [18] present a new integrated model in scheduling and routing by automated guided vehicles that focus on new methods of transportation. In some research, the types of products are more important than the types of transportation in practical models investigated in integrated modelling. Chen et al. [19] and Farahani et al. [3] propose a production and distribution scheduling for perishable food products. Liu et al. [20] present an integrated scheduling to improve the operation of production and delivery in ready-mixed concrete plants. Algorithms for solving these problems include approximation algorithms [21] and intelligent algorithms $[4,22,23]$. As mentioned, the type of product and transportation is important in practical cases and industries that is emphasized in this paper, as follows.

To the best of our knowledge, it seems that no research considers both permutation flow shop scheduling with due date and tardy cost in the production stage, and vehicle routing with consideration of deadline to satisfy customers. Few studies consider the transportation fleet in their models. This study is extended by assuming that the first stage of the supply chain is composed of $m$ machines as the flow shop environment in the production stage, and $v$ vehicles involved in designing vehicle routes for picking up and delivering finished goods in the second stage. It can be practical in real cases to serve a number of customers in various geographical zones.

\section{Problem description}

\subsection{Assumptions}

As mentioned previously, the scheduling of products and vehicles in a two-stage supply chain is investigated 
here. The first stage contains permutation flow shop scheduling with $m$ machines and $n$ jobs, and the second stage is composed of $v$ vehicles with different speeds and transportation capacities that transport $n$ jobs from the manufacturing company to $c$ customers distributed in various geographical zones. All vehicles are available upon completion of all jobs (i.e., $C_{\max }$ ).

The problem is described as follows. There is a set of jobs $(N=\{1,2, \cdots, n\})$ to be processed by a set of machines $(M=\{1,2, \cdots, m\})$ at the production stage, which is permutation flow shop scheduling. All jobs have to be processed in an identical order on a given set of machines. The processing of each job is continuous once the processing begins. After processing, the finished jobs need to be delivered by a set of vehicles, $V=\{1,2, \cdots, v\}$, which has a distinct capacity, $Q_{k}$, to a set of customers, $C=\{1,2, \cdots, c\}$, that aims to find a set of tours for several vehicles from a depot (i.e. manufacturer site) to a lot of customers. They return to the depot without exceeding capacity and deadline constraints. Each customer is visited only once by a single vehicle. Delay is not allowed, i.e. the jobs have to be delivered to the customers before the deadline. Moreover, when the last job terminates (i.e., makespan) after the due date, it will incur the tardy cost. The objective is to find an integrated production and distribution to minimize the sum of jobs' delivery cost and tardy cost that refers to the due date violation.

At the beginning of the horizon, customers require a set of jobs and send the requirements to the manufacturer. At the subsequent delivery stage, a fleet of capacitated vehicles delivers the finished jobs to the pre-specified customers. The capacity of the transporter is measured by a certain volume. Each customer is visited only once by a single vehicle. Only one vehicle is allowed to visit each customer.

\subsection{Mathematical model}

A mathematical formulation of the proposed Integrated Production-Distribution Scheduling (IPDS) problem is presented in this section. The parameters are as follows:

$\begin{array}{ll}n & \text { Number of jobs } \\ m & \text { Number of machines } \\ c & \text { Number of customers } \\ v & \text { Number of vehicles } \\ i & \text { Job index, } i=1,2, \cdots, n \\ p & \text { Job position index }, p=1,2, \cdots, n \\ r & \text { Machine index, } r=1,2, \cdots, m \\ j, l & \text { Customer index } \\ k & \text { Vehicle index } \\ v_{k} & \text { Speed of vehicle } k\end{array}$

$T_{r i} \quad$ Operation time of job $i$ on machine $r$

$d_{j l} \quad$ Distance between customer $j$ and customer $l$

$e_{i j} \quad$ Physical space of customer $j$ 's demand

$d d_{k} \quad$ Deadline for vehicle $k$ to deliver to customers

$Q^{k} \quad$ Capacity of vehicle $k$ for transporting job from manufacturing company to customers

$d u \quad$ Due date for production stage

cd Transportation cost per unit of distance

cp Violation due date cost in production stage

$M \quad$ A sufficiently large number

Variables of the model are as follows:

$\begin{array}{ll}B_{r j} & \begin{array}{l}\text { Start time of job } j \text { on machine } r \\ u_{j}\end{array} \\ r_{k} & \begin{array}{l}\text { Number of customers visited at } \\ \text { customer } j\end{array} \\ & \begin{array}{l}\text { Ready time of vehicle } k \text { representing } \\ \text { the latest completion time }\end{array} \\ c_{\max } & \begin{array}{l}\text { Maximum completion time of all jobs } \\ \text { (makespan) }\end{array} \\ z_{i j} & 1 \text { if job } i \text { in position } j \text { is processed; } \\ x_{j l}^{k} & \begin{array}{l}\text { zero otherwise } \\ 1 \text { if customer } l \text { is served after customer } \\ j \text { by vehicle } k ; \text { zero otherwise } \\ y\end{array} \\ & 1 \text { if } c_{\text {max }} \text { is after due date; zero } \\ & \text { otherwise }\end{array}$

Miller et al. [24] propose a mathematical programming formulation to prevent sub-tours in the vehicle routing problem. Kyparisis and Koulamas [25] propose a definition to consider the vehicle velocity and distance instead of time. All the above assumptions are used to develop the following model for the Integrated Production-Distribution Scheduling (IPDS) problem:

$$
\begin{array}{ll}
\operatorname{Min} c d \sum_{k=1}^{v} \sum_{l=0}^{c} \sum_{j=0}^{c} d_{l j} x_{l j}^{k}+c p . y\left(c_{\max }-d u\right), \\
\sum_{p=1}^{n} Z_{i p}=1 & 1 \leq i \leq n, \\
\sum_{i=1}^{n} Z_{i p}=1 & 1 \leq p \leq n, \\
B_{1 p}+\sum_{i=1}^{n} T_{1 i} Z_{i p}=B_{1, p+1} & 1 \leq p \leq n-1, \\
B_{11}=0, &
\end{array}
$$




$$
\begin{aligned}
& B_{r 1}+\sum_{i=1}^{n} T_{r i} Z_{i 1}=B_{r+1,1} \quad 1 \leq r \leq m-1, \\
& B_{r p}+\sum_{i=1}^{n} T_{r i} Z_{i p} \leq B_{r+1, p} \\
& 1 \leq r \leq m-1 \quad 2 \leq p \leq n, \\
& B_{r p}+\sum_{i=1}^{n} T_{r i} Z_{i p} \leq B_{r, p+1} \\
& 1 \leq p \leq n-1 \quad 2 \leq r \leq m, \\
& c_{\max }=B_{m n}+\sum_{i=1}^{n} T_{m i} Z_{i n} \\
& \sum_{k=1}^{v} \sum_{l=1}^{c} x_{j l}^{k}=1 \quad j=1,2, \cdots, c, \\
& \sum_{k=1}^{v} \sum_{j=1}^{c} x_{j l}^{k}=1 \quad l=1,2, \cdots, c, \\
& \sum_{l=0}^{c} x_{l h}^{k}-\sum_{j=0}^{c} x_{h j}^{k}=0 \\
& k=1,2, \cdots, v, \quad h=1,2, \cdots, c, \\
& \sum_{j=1}^{c} x_{0 j}^{k}=1, \quad k=1,2, \cdots, v, \\
& u_{j}+1 \leq u_{l}+c\left(1-x_{j l}^{k}\right) \quad k=1,2, \cdots, v, \\
& l=1,2, \cdots, c, \quad j=1,2, \cdots, c, \\
& \sum_{k=1}^{v} \sum_{j=1}^{c} x_{0 j}^{k}=v \\
& \sum_{k=1}^{v} \sum_{l=1}^{c} x_{l 0}^{k}=v \\
& \sum_{i=1}^{n} \sum_{j=0}^{c} \sum_{l=0}^{c} e_{i j} x_{l j}^{k} \leq Q^{k} \quad k=1,2, \cdots, v, \\
& r^{k} \geq c_{\max } \quad k=1,2, \cdots, v, \\
& r^{k}+\sum_{l=1}^{c} \sum_{j=0}^{c} d_{j l} / v_{k} x_{j l}^{k} \leq d d_{k} \quad k=1,2, \cdots, v, \\
& c_{\max } \geq d u-M(1-y), \\
& c_{\max } \leq d u+M y .
\end{aligned}
$$

The aim is to minimize the sum of delivery and tardy costs. Constraint sets (1) and (2) represent the assignment that guarantees to assign every job to one position and every position to one job. Constraint sets (3)-(5) ensure the permutation flow shop scheduling property in which there is no idle time on the first machine and the first job is processed on all machines without delay. Constraint set (6) guarantees that the start of each job on machine $r+1$ is not earlier than its finish time on machine $r$, which prevents the simultaneous processing of jobs on one machine. Constraint set (7) ensures that the job processes in position $p+1$ in the sequence do not start until the processing of the job in position $p$ on that machine is completed. Constraint set (8) represents makespan. Constraint sets (9) and (10) are common constraints for the vehicle routing problem that causes every customer to be serviced by only one vehicle. Constraint set (11) is the flow conservation constraint for each customer and when the vehicle serves the customer, it must depart the current customer to service the next ones. Constraint set (12) ensures that every vehicle must serve the customer once. Constraint set (13) prevents the sub tour that is disconnected to the depot. Constraint sets (14) and (15) confine the number of vehicles to serve the customers to $v$ vehicles. Constraint set (16) guarantees that the maximum flow in any arc leaving the root is equal to $Q^{k}$ and the maximum vehicle $k$ capacity is confined to $Q^{k}$. The relation of the production and distribution stages is important in the IPDS problem. Constraint set (17) focuses on integration of the production and distribution stages, which emphasizes the ready time of vehicles upon completion of all jobs. Constraint set (18) focuses on satisfying the deadline to serve the customer in order to increase customer satisfaction. Constraint sets (19) and (20) determine variable $y$ to get 1 or 0 that is dependent on makespan violation of the due date.

It should be mentioned that as the objective function contains non-linear terms, the mathematical model is a Mixed-Integer Non-Linear Programming (MINLP) model. The model could be solved with commercial software packages such as LINGO.

Lemma 1. The Integrated Production-Distribution Scheduling (IPDS) problem is NP-hard.

Proof. Chang and Lee [5] proved a problem with only one geographical zone, one manufacturer, and one vehicle as NP-hard. According to [26,27], the flow shop scheduling problem with $C_{\max }$ minimization objective is also NP-hard. As the IPDS problem is an extension of two NP-hard problems, i.e. the flow shop scheduling and vehicle routing problem, the integrated problem must be NP-hard in the strong sense. 
$\mathrm{NP}$ is not equal to $\mathrm{P}$ problems, so there is no algorithm to find global optima in polynomial computational time. Therefore, meta-heuristics or heuristics must be applied to solve large-scale problems within reasonable computation time. After introducing the particle swarm optimization algorithm, the improved algorithm, IPSO, is proposed in Section 4. In Section 5, it is validated by the generated test problems.

\section{Meta-heuristic algorithms}

Each of the integrated production and distribution scheduling problems is strongly NP-hard $[4,5]$. Therefore, an Improved Particle Swarm Optimization is proposed to deal with the proposed problem in this research. For validation of IPSO, a genetic algorithm emanated by [4] is applied.

\subsection{Improved Particle Swarm Optimization (IPSO)}

Various approaches have been proposed to solve scheduling problems. Among them, PSOs have been greatly adopted during recent years in practical domains $[28,29]$. The Particle Swarm Optimization (PSO) is a population-based optimization algorithm based on the benchmarking of social interactions such as bird flocking or fish school. It is developed by [30] in which the members of the whole population are maintained through the search procedure, so that information is socially shared among individuals to guide the search towards the best position in the search space. PSO is an evolutionary algorithm, which is initialized with a population (swarm) of random solutions (particles) that fly in the search space. It searches for optima by updating generations. Individuals or potential solutions are named particles. They fly in the problem space with a velocity dynamically updated according to the flying experiences of every particle and the whole population.

The PSO algorithm has been successfully used for various applications such as neural network training, task assignment, supplier selection, ordering problem, permutation flow shop sequencing problems, lot sizing problem, and single machine total weighted tardiness problems $[31,32]$. The advantages of a PSO algorithm are observable not only in the wide applications, but also in the simple structures, immediate accessibility for practical applications, ease of implementation, speed to get the solutions, and robustness.

The basic elements of the PSO algorithm are summarized as follows:

- Particle: $X_{i}^{t}$ is the $i$ th particle at iteration $t$ with a $D$-dimensional vector, which is located at $X_{i}^{t}=$ $\left(x_{i 1}^{t}, x_{i 2}^{t}, \cdots, x_{i D}^{t}\right)$ in the searching space;

- Population: Consists of $P$ particles with $D$ dimensions in the swarm, iteratively;
- Particle velocity: The $i$ th particle's velocity is also a $D$-dimensional vector as $V_{i}^{t}=v_{i 1}^{t}, v_{i 2}^{t}$, $\cdots, v_{i D}^{t}$, which is updated iteratively;

- Inertia weight and acceleration coefficients: $\omega$ is a parameter to control the impact of previous velocities on the current velocity. $c_{1}$ and $c_{2}$ are constant parameters called acceleration coefficients to control the maximum step size that the particle can carry out;

- Personal best: The best position of the $i$ th particle with the best fitness value until iteration $t$ is $P_{i}^{t}=\left(p_{i 1}^{t}, p_{i 2}^{t}, \cdots, p_{i D}^{t}\right)$ that means the best position associated with the best fitness value of the particles so far. It is updated iteratively for each particle;

- Global best: The best position of the population obtained so far in the whole swarm is $P_{g}^{t}=$ $\left(p_{g}^{t}, p_{g}^{t}, \cdots, p_{g}^{t}\right)$ at iteration $t$

- Termination criterion: It defines a condition to stop searching the solution space. It is optional according to the max generation or CPU time.

The velocity of each particle based on the current velocity, the best experience of the particle, and that of the entire population are updated as follows:

$$
v_{i}^{t}=\omega \cdot v_{i d}^{t-1}+c_{1} \cdot r_{1}\left(p_{i d}^{t}-x_{i d}^{t}\right)+c_{2} \cdot r_{2}\left(p_{g d}^{t}-x_{i d}^{t}\right) .
$$

Eq. (1) describes the velocity of a particle at iteration $t$. It is determined by the previous velocity of the particle, the cognition part, and the social part. $c_{1}$ is the cognition factor, $c_{2}$ is the social learning factor, and $\omega$ is inertia weight to control the impact of previous velocities on the current velocity, calculated in Eq. (2). The position of each particle is updated in each iteration in Eq. (3):

$$
\begin{aligned}
& \omega=\frac{2}{\left|2-\varphi-\sqrt{\varphi^{2}-4 \varphi}\right|} \\
& \varphi=\varphi_{1}+\varphi_{2}>4 \\
& c_{1}=\omega \varphi_{1} \\
& c_{2}=\omega \varphi_{2} \\
& x_{i d}^{t}=x_{i d}^{t-1}+v_{i d}^{t} .
\end{aligned}
$$

In order to control excessive flying of particles outside the search space, the velocity values are confined to the range $\left[-v_{\max }, v_{\max }\right]$.

\subsubsection{Proposed IPSO algorithm}

In this section, a particle swarm optimization algorithm named Improved Particle Swarm Optimization (IPSO) algorithm is proposed. The procedure of the IPSO is 


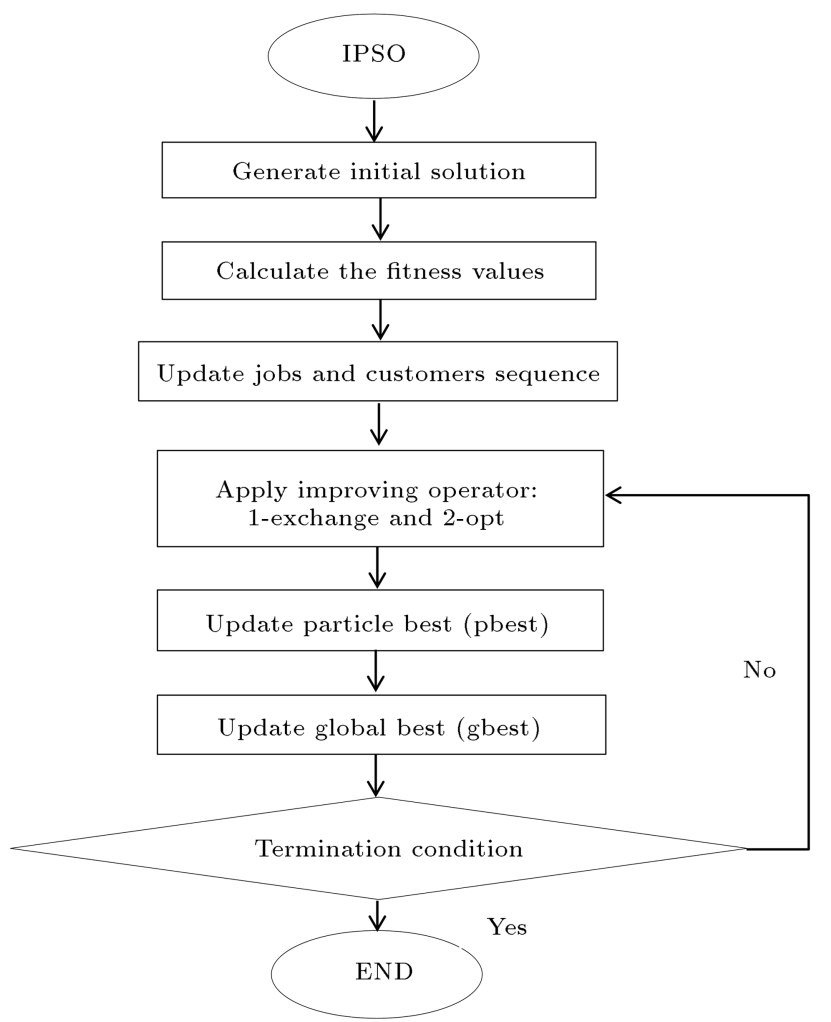

Figure 1. Process flow of proposed IPSO.

shown in Figure 1. For this purpose, among many different kinds of neighbourhood structures in the literature, a local improvement procedure, namely (1exchange, 2-opt), is added to improve the solution's quality. It is randomly selected for each iteration to extend solution search. The following two types are considered to improve solution quality and widen search space:

- 1-exchange: Select two tours; then, omit one customer in one of two tours and add it to the other tour;

- 2-opt: Select two tours; then, interchange two customers of one tour with two of the other.

When a particle is going to stagnate, the improving operators are used to search its neighbourhood to prevent premature convergence. The improving structures can influence quality of the solution. Numerical results show how these procedures prevent the algorithm from getting stuck in a local optimum.

\subsection{Computational procedure}

The complete computational procedure of the IPSO is summarized as follows:

\section{- Step 1: Initialization:}

- Initialize $P$ particles randomly as a population with $(D=n+c+v-1)$-dimensions; $n, c$, $v$, respectively refer to the numbers of jobs, customers, and vehicles. In the production stage, customers are randomly assigned to a definite number of $v$ vehicles. There is a capacity violation cost in order to guarantee the vehicle capacity constraint and time violation cost to satisfy the deadline constraint by minimizing violation costs. In the distribution stage, the permutation of jobs is considered in the initial population with consideration of better fitness value (lower objective function). First, calculate each particle's fitness value of the initialization population and, then, rank them. Choose the smaller one that is $P_{i}^{t}$. Choose the particle with the best fitness value of the whole population as $P_{g}^{t}$;

- Set $t=1$; generate the position of particle $i, X_{i}^{1}(i=1,2, \cdots, p)$ randomly, where $X_{i}^{1}=$ $\left(x_{i 1}^{1}, x_{i 2}^{1}, \cdots, x_{i D}^{1}\right)$ for the whole population;

- Generate velocity of particle $i, V_{i}^{1}(i=1,2, \cdots, p)$ randomly, where $V_{i}^{1}=v_{i 1}^{1}, v_{i 2}^{1}, \cdots, v_{i D}^{1}$ for the whole population;

- Compute the performance measurement, i.e. the total delivery and tardy cost, and set it as the fitness value $f_{i}^{1}$ of $x_{i}^{1}(i=1,2, \cdots, p)$;

- For each particle in the swarm, $P_{i}^{1}=\left(p_{i 1}^{1}=\right.$ $\left.x_{i 1}^{1}, p_{i 2}^{1}=x_{i 2}^{1}, \cdots, p_{i D}^{1}=x_{i D}^{1}\right)$ for $i=1,2, \cdots, p ;$

- Find the best fitness value in the population $P_{g}^{1}=$ $\min \left(f_{i}^{1}\right)$ for $i=1,2, \cdots, p$.

- Step 2: Update iteration:

- $t=t+1$.

- Step 3: Apply improving operator:

- Apply one of two improving operators randomly at each iteration to search the solution space widely and achieve various solutions.

- Step 4: Update $P_{i}^{t}$ and $P_{g}^{t}$ :

- For $i=1,2, \cdots, p$ calculate the fitness value; if $f_{i}^{t}<P_{i}^{t-1}$, put $P_{i}^{t}=f_{i}^{t}$; otherwise, $P_{i}^{t}=P_{i}^{t-1}$;

- For $i=1,2, \cdots, p$ calculate fitness value; find the minimum fitness value in the swarm; if $\min \left(f_{i}^{t}\right)<$ $P_{g}^{(t-1)}$, put $P_{g}^{t}=\min \left(f_{i}^{t}\right)$; otherwise, $P_{g}^{t}=P_{g}^{t-1}$.

- Step 5: Update velocity:

- $v_{i}^{t}=\omega \cdot v_{i d}^{t-1}+c_{1} \cdot r_{1}\left(p_{i d}^{t}-x_{i d}^{t}\right)+c_{2} \cdot r_{2}\left(p_{g d}^{t}-x_{i d}^{t}\right)$; $\varphi_{1}$ and $\varphi_{2}$ are equal to $2.05 ; r_{1}$ and $r_{2}$ are random numbers uniformly distributed in $[0,1]$, and $c_{1}$ and $c_{2}$ are random numbers in $[0,2]$.

- Step 6: Update position

- $x_{i d}^{t}=x_{i d}^{t-1}+v_{i d}^{t}$.

- Step 7: Termination criterion

- The termination criterion is determined when the number of iterations is achieved by the max generation; otherwise, go to Step 2. 


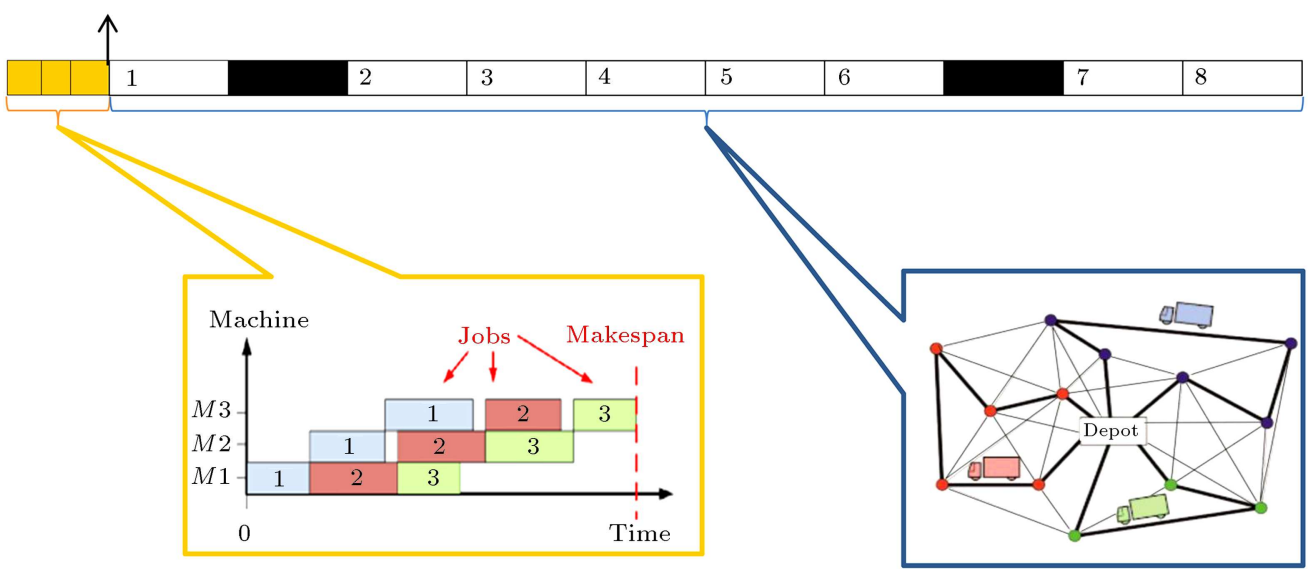

Figure 2. The particle encoded as the number of jobs and customers.

\subsection{Solution representation in an illustrative example}

In an integrated production and distribution scheduling problem, the information of both problems is stored in one particle instead of defining two separate particles. Hence, the encoding scheme contains real numbers. Each particle is consisted to have $(n+c+v-1)$ dimensions, in which $n, c$, and $v$ are, respectively, the numbers of jobs, customers, and vehicles.

In this section, an illustrative example has been included to show the efficiency of the proposed approach. After completion of jobs $\left(C_{\max }\right)$ in the production stage, which contains permutation flow shop scheduling, vehicles deliver customers' orders with restrictions of vehicles capacity and deadline constraint. An instance of the problem with three jobs, eight customers, and three vehicles is given in Figure 2 as an example of the encoding integrated problem. A particle in this encoding system is a feasible sequence of jobs that are assigned to machines in spite of the sequence of customers that are assigned to vehicles with respect to vehicle capacity.

\section{Computational results}

In this section, the computational experiments are performed to test the performance of the proposed algorithm. The IPSO algorithm is tested on different scale instances and compared with the recently proposed efficient algorithms. As there are no benchmark solutions to compare with the solution of the proposed algorithm, for small-size and moderate-size instances, the IPSO algorithm is compared with the optimal solution calculated by a commercial optimization software, LINGO 13.0. For large-size instances, it is compared with the genetic algorithm of the similar problem [4]. All the algorithms in this paper are coded in MATLAB 8.3. They are implemented on a computer with Intel Core 2Duo 2.5 GHZ and $3 \mathrm{~GB}$ RAM.

\subsection{Test problems generation}

Test problems are randomly generated as follows. 25 random instances for each problem size are created based on the following parameter settings:

- The job processing time on the machine is randomly generated from the uniform distribution in the range of $[1,99]$;

- The volumes of different jobs are randomly generated from the uniform distribution in the range of $[1,10]$;

- The distance between different customers is randomly generated from the uniform distribution in the range of $[1,100]$;

- The vehicle capacity is randomly generated from the uniform distribution in the range of $\left[\sum_{j=1}^{c}\right.$ $\left.\sum_{i=1}^{n} e_{i j} / v, \sum_{j=1}^{c} \sum_{i=1}^{n} e_{i j}\right]$;

- In this paper, a new and practical range is applied with consideration of velocity and distance, the deadlines of which are randomly generated from the uniform distribution in the range of $\left[\alpha \sum_{r=1}^{m} \sum_{i=1}^{n} T_{r i}, \lambda\left(T_{\min }+T_{\max }\right) / 2+\left(d_{\min }+\right.\right.$ $\left.\left.d_{\max }\right) / 2 v\right]$, where $T_{\min }$ and $T_{\max }$ are minimum and maximum job processing times, respectively, and $d_{\text {min }}$ and $d_{\max }$ are the minimum and maximum distances, respectively in which $\alpha=0.2$ and $\lambda=$ 1.5 by numerical experiments. It is different from Pundoor and Chen's [13] research;

- The due dates are randomly generated from the uniform distribution in range of $[\overline{d u}(1-R / 2), \overline{d u}(1+$ $R / 2)$ ] that $\overline{d u}=\sum_{r=1}^{m} \sum_{i=1}^{n} T_{r i}(1-\tau), R=0.6$, $\tau=0.2[33]$.

Problems are classified into three groups of small, moderate, and large sizes in order to compare exact procedures, IPSO, and GA, for each test problem that is described in the next section. 
Table 1. Results of random instances with small sizes.

\begin{tabular}{|c|c|c|c|c|c|c|c|c|c|}
\hline \multirow{2}{*}{$\begin{array}{c}\text { Instance } \\
\text { no. }\end{array}$} & \multicolumn{4}{|c|}{ Size } & \multicolumn{2}{|c|}{ IPSO } & \multicolumn{2}{|c|}{ LINGO } & \multirow[b]{2}{*}{ PD } \\
\hline & Machine & Job & Vehicle & Customer & Value & $\begin{array}{c}\text { CPUT } \\
(\mathrm{s})\end{array}$ & Value & $\begin{array}{c}\text { CPUT } \\
(\mathrm{s})\end{array}$ & \\
\hline 1 & 2 & 5 & 2 & 6 & 29493 & 22 & 29493 & 4 & 0 \\
\hline 2 & 2 & 10 & 3 & 6 & 38736 & 19 & 38736 & 17 & 0 \\
\hline 3 & 2 & 15 & 3 & 6 & 39937 & 20 & 39937 & 42 & 0 \\
\hline 4 & 5 & 5 & 2 & 6 & 32967 & 19 & 33517 & 12 & 0 \\
\hline 5 & 5 & 15 & 2 & 6 & 42337 & 20 & 42234 & 60 & 0.002 \\
\hline 6 & 2 & 5 & 3 & 7 & 30043 & 21 & 29993 & 38 & 0.001 \\
\hline 7 & 2 & 10 & 3 & 7 & 32586 & 20 & 32586 & 27 & 0 \\
\hline 8 & 2 & 15 & 3 & 7 & 32837 & 21 & 32837 & 51 & 0 \\
\hline 9 & 5 & 5 & 2 & 7 & 29467 & 19 & 29467 & 15 & 0 \\
\hline 10 & 5 & 15 & 2 & 7 & 38101 & 22 & 38184 & 52 & 0 \\
\hline 11 & 2 & 5 & 3 & 8 & 29943 & 17 & 32443 & 23 & 0 \\
\hline 12 & 2 & 10 & 3 & 8 & 39886 & 17 & 39886 & 160 & 0 \\
\hline 13 & 2 & 15 & 3 & 8 & 40154 & 21 & 40137 & 129 & 0.0004 \\
\hline 14 & 5 & 5 & 2 & 8 & 34467 & 21 & 34467 & 107 & 0 \\
\hline 15 & 5 & 15 & 2 & 8 & 43984 & 17 & 43834 & 136 & 0.003 \\
\hline 16 & 2 & 5 & 3 & 9 & 30143 & 17 & 33843 & 116 & 0 \\
\hline 17 & 2 & 10 & 3 & 9 & 40236 & 19 & 40236 & 160 & 0 \\
\hline 18 & 2 & 15 & 3 & 9 & 40637 & 20 & 40487 & 92 & 0.004 \\
\hline 19 & 5 & 5 & 2 & 9 & 33417 & 19 & 33417 & 72 & 0 \\
\hline 20 & 2 & 5 & 3 & 10 & 24983 & 19 & 24943 & 59 & 0.001 \\
\hline 21 & 2 & 10 & 2 & 10 & 34236 & 16 & 33936 & 67 & 0.009 \\
\hline 22 & 2 & 15 & 2 & 10 & 37187 & 17 & 37237 & 153 & 0 \\
\hline 23 & 5 & 5 & 2 & 10 & 28417 & 21 & 28317 & 37 & 0.003 \\
\hline 24 & 5 & 10 & 2 & 10 & 34759 & 20 & 34759 & 90 & 0 \\
\hline 25 & 5 & 15 & 2 & 10 & 43034 & 16 & 43147 & 548 & 0 \\
\hline Average & & & & & & 19.2 & & 83 & 0.0009 \\
\hline
\end{tabular}

\subsection{Validation and verification of the IPSO}

In this section, the outcomes of the model in the exact procedure, IPSO, and GA for each test problem, in the way of randomized block design [34], are reported. The tables consist of three parts: the objective function, running time (CPUT), and percentage deviation. The indicator PD (Percentage Deviation) is defined as $\mathrm{PD}=100 \times($ IPSOs - LINGOs $) /$ LINGOs to assess the quality of the obtained solution. It measures the amount of improvement in terms of the objective function. In the PD formula, LINGOs and IPSOs represent the LINGO solution and IPSO solution, respectively. It should be mentioned that LINGO finds the optimal solution for all small-size instances.

For comparison, the MINLP model is solved with the mathematical software LINGO 13.0. It is used to exactly solve the model with small-size instances. Since the complexity of the problem is categorized in the NP-hard class, only small-size problems can be solved. All PD percentages and the total average of the small-size problems shown in Table 1 are less than $0.9 \%$. The average running time of the IPSO is less than that of the optimal approach. For moderate-size problems, LINGO returns a local optimal solution in a limitation of 2000 seconds for running time. Table 2 shows the solutions of the proposed IPSO algorithm, which are better than the local optimal solutions found by LINGO. The gap of the local solution is $9.5 \%$ on average for all moderate-size instances. For moderatesize problems, the $\mathrm{PD}$ formula is rewritten as $\mathrm{PD}=$ $100 \times \max (0$, IPSOs - LINGOs $) /$ LINGOs.

It is shown that the IPSO runs much faster than the LINGO solver. Although the LINGO solver finds the optimal solution, the computational time of LINGO grows exponentially as the instance size increases. Moreover, the IPSO cannot obtain optimal solutions for moderate sizes, but can find locally optimal solutions in the limitation time of 2000 seconds. 
Table 2. Results of random instances with moderate sizes.

\begin{tabular}{|c|c|c|c|c|c|c|c|c|c|}
\hline \multirow{2}{*}{$\begin{array}{c}\text { Instance } \\
\text { no. }\end{array}$} & \multicolumn{4}{|c|}{ Size } & \multicolumn{2}{|c|}{ IPSO } & \multicolumn{2}{|c|}{ LINGO } & \multirow[b]{2}{*}{ PD } \\
\hline & Machine & Job & Vehicle & Customer & Value & $\begin{array}{c}\text { CPUT } \\
(\mathrm{s})\end{array}$ & Value & $\begin{array}{c}\text { CPUT } \\
(\mathrm{s})\end{array}$ & \\
\hline 1 & 2 & 100 & 10 & 10 & 118481 & 21 & 122976 & 2000 & 0 \\
\hline 2 & 2 & 50 & 20 & 10 & 96522 & 23 & 96706 & 2000 & 0 \\
\hline 3 & 2 & 50 & 10 & 20 & 89117 & 22 & 96535 & 2000 & 0 \\
\hline 4 & 5 & 10 & 10 & 100 & 169617 & 29 & 141113 & 2000 & 0.20 \\
\hline 5 & 4 & 20 & 10 & 50 & 98768 & 25 & 135901 & 2000 & 0 \\
\hline 6 & 4 & 200 & 10 & 10 & 214359 & 29 & 218871 & 2000 & 0 \\
\hline 7 & 4 & 50 & 40 & 10 & 125039 & 26 & 122600 & 2000 & 0.02 \\
\hline 8 & 5 & 100 & 10 & 20 & 150885 & 26 & 133666 & 2000 & 0.12 \\
\hline 9 & 5 & 200 & 5 & 20 & 220857 & 28 & 211838 & 2000 & 0.04 \\
\hline 10 & 6 & 100 & 5 & 40 & 159283 & 20 & 121982 & 2000 & 0.30 \\
\hline 11 & 7 & 100 & 4 & 50 & 173507 & 29 & 131489 & 2000 & 0.21 \\
\hline 12 & 7 & 20 & 20 & 50 & 127811 & 26 & 137781 & 2000 & 0 \\
\hline 13 & 7 & 80 & 5 & 50 & 149301 & 22 & 113730 & 2000 & 0.31 \\
\hline 14 & 7 & 20 & 10 & 100 & 19727 & 23 & 73188 & 2000 & 0 \\
\hline 15 & 6 & 60 & 10 & 50 & 164775 & 22 & 174470 & 2000 & 0 \\
\hline 16 & 5 & 100 & 10 & 30 & 167996 & 21 & 181159 & 2000 & 0 \\
\hline 17 & 6 & 30 & 10 & 100 & 216093 & 24 & 171509 & 2000 & 0.25 \\
\hline 18 & 5 & 50 & 10 & 60 & 164422 & 22 & 158756 & 2000 & 0.03 \\
\hline 19 & 5 & 60 & 10 & 50 & 158729 & 22 & 164456 & 2000 & 0 \\
\hline 20 & 6 & 30 & 20 & 50 & 117182 & 22 & 171450 & 2000 & 0 \\
\hline 21 & 3 & 50 & 40 & 15 & 128993 & 22 & 143768 & 2000 & 0 \\
\hline 22 & 4 & 150 & 10 & 20 & 188416 & 22 & 162631 & 2000 & 0.16 \\
\hline 23 & 5 & 30 & 10 & 100 & 211993 & 23 & 178145 & 2000 & 0.19 \\
\hline 24 & 6 & 40 & 10 & 75 & 174108 & 22 & 141551 & 2000 & 0.23 \\
\hline 25 & 6 & 20 & 20 & 75 & 159411 & 24 & 120766 & 2000 & 0.32 \\
\hline Average & & & & & 23.76 & & & 2000 & 0.095 \\
\hline
\end{tabular}

The superiority of the suggested IPSO is examined by analysis of variance and Tukey pairwise comparisons [34]. The statistical model presented in Eq. (4) is the same for both response variables, i.e. objective function and running time. Consequently, $Y_{i j}$ can be the objective function or time duration of the $i$ th test problem solved by the $j$ th algorithm (LINGO, IPSO, or GA). The size of the test problem is considered as the block effect $\left(p_{i}\right)$ in $I$ levels and the algorithm is determined as the main factor $\left(a_{j}\right)$ in $J$ levels. The statistical model is as follows:

$$
\begin{aligned}
Y_{i j} & =\mu+p_{i}+a_{j}+\varepsilon_{i j}, \\
i & =1,2, \cdots, I, \quad j=1,2, \cdots, J .
\end{aligned}
$$

In order to show that the proposed IPSO converges on the optimal solution in a polynomial time for solving small-size and moderate-size test problems, the results of Tables 1 and 2 are utilized. As mentioned before, the executions are based on a randomized block design with $I=50$ and $J=2$.

Tables 3 and 4 report the analysis results of variance obtained by a statistical software package, MINITAB 17. Moreover, the results of the Tukey test are reported in Tables 5 and 6 . According to Table 3, the equivalence hypothesis of LINGO and IPSO solutions in terms of objective function is not rejected. Furthermore, based on Table 5, both LINGO and IPSO algorithms are categorized in the same group. As a result, the convergence of IPSO on the optimal solution, i.e., the verification of IPSO, is statistically proven.

Tables 4 and 6 represent results of the ANOVA and Tukey test for CPU times, respectively. As the $P$-value of Table 4 for the algorithm is about zero, LINGO and IPSO solutions are different in terms of running times. With different categories of the Tukey test in Table 5, it is proven that the running 
Table 3. Results of comparing exact solutions and IPSO solutions with respect to objective function.

\begin{tabular}{lccccc}
\hline \multicolumn{1}{c}{ Source } & DF & SS & MS & $\boldsymbol{F}$-value & $\boldsymbol{P}$-value \\
\hline Algorithm & 1 & 174952471 & 174952471 & 0.88 & 0.354 \\
Problem & 49 & 387824000000 & 7914775129 & 39.64 & 0.000 \\
Error & 49 & 9784033151 & 199674146 & & \\
Total & 99 & 397783000000 & & & \\
\hline
\end{tabular}

Table 4. Results of comparing exact solutions and IPSO solutions with respect to running time.

\begin{tabular}{lccccc}
\hline \multicolumn{1}{c}{ Source } & DF & SS & MS & $\boldsymbol{F}$-value & $\boldsymbol{P}$-value \\
\hline Algorithm & 1 & 26208553 & 26208553 & 56.29 & 0.000 \\
Problem & 49 & 23030099 & 470002 & 1.01 & 0.487 \\
Error & 49 & 22813714 & 465586 & & \\
Total & 99 & 72052366 & & & \\
\hline
\end{tabular}

Table 5. Tukey results of comparing exact solutions and IPSO solutions with respect to objective function.

\begin{tabular}{cccc}
\hline Tukey grouping & Mean & $\boldsymbol{N}$ & Algorithm \\
\hline A & 92947.6 & 50 & LINGO \\
A & 90302.2 & 50 & IPSO \\
\hline
\end{tabular}

Table 6. Tukey results of comparing exact solutions and IPSO solutions with respect to running time.

\begin{tabular}{cccc}
\hline Tukey grouping & Mean & $\boldsymbol{N}$ & Algorithm \\
\hline A & 1045.34 & 50 & LINGO \\
B & 21.45 & 50 & IPSO \\
\hline
\end{tabular}

time of IPSO is much less than that of the LINGO solver.

As LINGO cannot solve large-size problems, for these instances, the proposed IPSO algorithm is compared with the genetic algorithm proposed by [4] for the similar integrated scheduling problem. This is done to assess the efficiency of the proposed algorithm in solving real cases. The $\mathrm{PD}$ indicator is not defined here because the optimal solutions are not available. Table 7 presents the results of IPSO and GA in terms of the objective function and the CPU time. The IPSO algorithm is capable of generating solutions with good quality and within a reasonable amount of CPU time, which is less than that in the GA algorithm on average.

Using the ANOVA procedure and Tukey test, both IPSO and GA are compared with each other. Results of the statistical model with MINITAB 17 , where $I=25$ and $J=2$, are reported in Tables 811. According to Tables 7 and 9 , the algorithms are significantly different in terms of the solution's quality. Therefore, IPSO is greatly superior to GA.

In addition to the objective function, the comparison made between the two algorithms in terms of CPU time is reported in Tables 9 and 11. It shows that running times of both IPSO and GA do not differ significantly because the $P$-value is larger than $5 \%$ and they are in the same category of the Tukey test. However, this comparison is not worth mentioning because IPSO and GA are both meta-heuristics which solve problems in polynomial time.

From the above test results, it is found that the proposed IPSO algorithm can solve IPDS efficiently, providing reliable solutions. It is more effective and better than the other compared algorithms, i.e. GA and LINGO software. The efficiency of the IPSO makes it suitable for solving real cases, which are normally large in scale.

\subsection{Managerial implication}

This paper discusses the practical implications of managerial decisions to integrate production and distribution scheduling. The focus of this study is to show the practical implication of the integrated model to consider simultaneous flowshop scheduling and vehicle routing decisions. The integrated scheduling problems studied in this paper are based on the models for production and distribution that are applied to a wide range of practical applications. The integrated approach prevents decrease in product quality, expiration before delivery, extra expenses, and lack of customer satisfaction. It is practical in many industries involving make-to-order or time-sensitive (e.g., perishable, dairy) products, where finished orders are often delivered to customers immediately or shortly after the production to restrict quality reduction.

\section{Conclusion and future research}

This paper has dealt with practice-oriented integrated production and distribution scheduling. IPDS focuses on products with a short lifespan that include flowshop scheduling decisions in a single plant, a fleet of limitedcapacity trucks for delivering customers' demand with consideration of vehicle routing, and a number of 
Table 7. Results of random instances with large sizes.

\begin{tabular}{|c|c|c|c|c|c|c|c|c|}
\hline \multirow{2}{*}{$\begin{array}{c}\text { Instance } \\
\text { no. }\end{array}$} & \multicolumn{4}{|c|}{ Size } & \multicolumn{2}{|c|}{ IPSO } & \multicolumn{2}{|c|}{ GA } \\
\hline & Machine & Job & Vehicle & Customer & Value & $\begin{array}{c}\text { CPUT } \\
(\mathrm{s})\end{array}$ & Value & $\begin{array}{c}\text { CPUT } \\
(\mathrm{s})\end{array}$ \\
\hline 1 & 200 & 20 & 20 & 200 & 648781 & 69 & 937723 & 73 \\
\hline 2 & 200 & 30 & 30 & 200 & 697987 & 79 & 1052365 & 68 \\
\hline 3 & 200 & 50 & 50 & 200 & 773656 & 121 & 1151122 & 150 \\
\hline 4 & 200 & 20 & 50 & 200 & 815652 & 120 & 1283420 & 109 \\
\hline 5 & 200 & 30 & 50 & 200 & 761005 & 110 & 1353470 & 88 \\
\hline 6 & 200 & 50 & 50 & 300 & 841468 & 126 & 1064971 & 139 \\
\hline 7 & 200 & 50 & 30 & 500 & 1058764 & 129 & 1557706 & 116 \\
\hline 8 & 200 & 40 & 50 & 400 & 1006698 & 152 & 1186959 & 181 \\
\hline 9 & 300 & 20 & 20 & 300 & 964474 & 90 & 1363511 & 153 \\
\hline 10 & 300 & 30 & 30 & 300 & 1080526 & 106 & 1279986 & 200 \\
\hline 11 & 300 & 50 & 50 & 200 & 968308 & 111 & 1649322 & 86 \\
\hline 12 & 300 & 20 & 50 & 300 & 1039654 & 138 & 1299667 & 177 \\
\hline 13 & 300 & 30 & 50 & 300 & 1146759 & 138 & 1533116 & 121 \\
\hline 14 & 300 & 50 & 50 & 300 & 1151689 & 136 & 1476345 & 150 \\
\hline 15 & 300 & 50 & 30 & 500 & 1255513 & 137 & 1484446 & 103 \\
\hline 16 & 300 & 40 & 50 & 400 & 1250807 & 164 & 1537098 & 99 \\
\hline 17 & 500 & 20 & 20 & 500 & 1700525 & 130 & 1932005 & 160 \\
\hline 18 & 500 & 30 & 30 & 500 & 1715614 & 157 & 2033918 & 188 \\
\hline 19 & 500 & 50 & 50 & 400 & 1688019 & 183 & 2245663 & 180 \\
\hline 20 & 500 & 20 & 50 & 500 & 1980044 & 210 & 2663903 & 199 \\
\hline 21 & 500 & 30 & 50 & 500 & 1809463 & 201,4 & 2483166 & 190 \\
\hline 22 & 500 & 50 & 50 & 500 & 1788781 & 211 & 2311044 & 201 \\
\hline 23 & 500 & 50 & 30 & 500 & 1755100 & 157 & 2250077 & 190 \\
\hline 24 & 500 & 40 & 50 & 400 & 1733551 & 174 & 2818420 & 189 \\
\hline 25 & 400 & 50 & 50 & 400 & 1378299 & 174 & 2913846 & 178 \\
\hline Average & & & & & & 132.8 & & 147.5 \\
\hline
\end{tabular}

Table 8. Results of comparing exact solutions and IPSO solutions with respect to the objective function.

\begin{tabular}{lccccc}
\hline \multicolumn{1}{c}{ Source } & DF & SS & MS & $\boldsymbol{F}$-value & $\boldsymbol{P}$-value \\
\hline Algorithm & 1 & 2809460000000 & 2809460000000 & 61.48 & 0.000 \\
Problem & 24 & 11278000000000 & 469918000000 & 10.28 & 0.000 \\
Error & 24 & 1096650000000 & 45693786706 & & \\
Total & 49 & 15184100000000 & & & \\
\hline
\end{tabular}

Table 9. Results of comparing exact solutions and IPSO solutions with respect to the CPU time.

\begin{tabular}{lccccc}
\hline \multicolumn{1}{c}{ Source } & DF & SS & MS & $\boldsymbol{F}$-value & $\boldsymbol{P}$-value \\
\hline Algorithm & 1 & 545.2 & 545.2 & 1.00 & 0.326 \\
Problem & 24 & 67577.0 & 2815.7 & 5.19 & 0.000 \\
Error & 24 & 13023.5 & 542.6 & & \\
Total & 49 & 81145.7 & & & \\
\hline
\end{tabular}

customers with defined locations and demands. The goal was to design routes that serve all customers within each trip and to schedule production in order to minimize the total tardy and delivery costs while fulfilling the vehicle capacity and deadline for each trip in order to meet lifespan constraints. In the perishable products industry, costs are perhaps the most prominent feature. They are strongly influenced by the two interrelated stages of production and distribution. In this paper, a scheduling problem in a two-stage supply chain environment is proposed with the objective function of minimizing the sum of delivery and tardy 
Table 10. Tukey results of IPSO and GA with respect to the objective function.

\begin{tabular}{cccc}
\hline Tukey grouping & Mean & $\boldsymbol{N}$ & Algorithm \\
\hline A & 1714531 & 25 & GA \\
B & 1240445 & 25 & IPSO \\
\hline
\end{tabular}

Table 11. Tukey results of IPSO and GA with respect to the CPU time.

\begin{tabular}{cccc}
\hline Tukey grouping & Mean & $\boldsymbol{N}$ & Algorithm \\
\hline A & 147.536 & 25 & GA \\
B & 140.932 & 25 & IPSO \\
\hline
\end{tabular}

costs. The production and distribution scheduling is integrated into flow shop environment and vehicle routing. This model is still a good representation of the real-world cases such as the dairy product industry. After presenting the IPDS problem as a mixed integer nonlinear programming model, since the problem is NP-hard in the strong sense, an Improved Particle Swarm Optimization (IPSO) algorithm was proposed to schedule the integrated problem. 1-exchange and 2-opt improving operators enhanced quality of the solution in IPSO. Since this is the first study of this problem, a new data set was generated.

Results of the numerical survey indicated decreasing costs and, as a result, increasing customer satisfaction and product quality. All of the 25 smallsize instances were optimally solved by the proposed IPSO. The mean relative deviation from the optimum of $0.9 \%$ was close to zero. The gap of the local solution was $9.5 \%$ on average for all moderate-size instances. The superiority of the suggested IPSO was examined by analysis of variance and Tukey pairwise comparisons. As a result, the convergence of IPSO on the optimal solution, i.e. the verification of IPSO, was statistically proven. Moreover, for large-size instances, the proposed IPSO algorithm was compared with the genetic algorithm proposed by [4] for the similar integrated scheduling problem. Using the ANOVA procedure and Tukey test, both IPSO and GA were compared. Results of the statistical model reported that the efficiency of the IPSO made it suitable for solving real cases, which were normally large in scale. An investigation of large-size instances indicated that the IPSO was able to provide relatively good results within an acceptable computation time.

Future research is needed to investigate an integrated scheduling problem where the machine configurations and distribution are more complex, such as batch scheduling, two-stage vehicle routing, or multi plants which are distributed in various geographic regions. Integration of different decision levels, like tactical or strategic, helps in optimizing the whole supply chain. Tightening the gap between theory and practical applications would be highly worthwhile for more research.

\section{References}

1. Schmid, V., Doerner, K.F. and Laporte, G. "Rich routing problems arising in supply chain management", European Journal of Operational Research, 228, pp. 435-448 (2013).

2. Diaz-Madronero, M., Peidro, D. and Mula, J. "A review of tactical optimization models for integrated production and transport routing planning decisions", Computers \& Industrial Engineering, 88, pp. 518-535 (2015).

3. Farahani, P., Grunow, M. and Gunther, H.O. "Integrated production and distribution planning for perishable food products", Flexible Service Manufacturing Journal, 24(1), pp. 28-51 (2012).

4. Ullrich, C.A. "Integrated machine scheduling and vehicle routing with time windows", European Journal of Operational Research, 227(1), pp. 152-165 (2013).

5. Chang, Y.C. and Lee, C.Y. "Machine scheduling with job delivery coordination", European Journal of Operational Research, 158(2), pp. 470-487 (2004).

6. Chen, Z.L. "Integrated production and outbound distribution scheduling: Review and extensions", Operations Research, 58(1), pp. 130-148 (2010).

7. Chen, H.K., Hsueh, C.F. and Chang, M.S. "Production scheduling and vehicle routing with time windows for perishable food products", Computers \& Operation Research, 36(7), pp. 2311-2319 (2009).

8. Buer, M.G.V., Woodruff, D.L. and Olson, R.T. "Solving the medium newspaper production/distribution problem", European Journal of Operational Research, 115(2), pp. 237-253 (1999).

9. Wang, Q., Batta, R. and Szczerba, R.J. "Sequencing the processing of incoming mail to match an outbound truck delivery schedule", Computers and Operations Research, 32(7), pp. 1777-1791 (2005).

10. Devapriya, P., Ferrell, W. and Geismar, N., Optimal Fleet Size of an Integrated Production and Distribution Scheduling Problem for a Perishable Product, Working Paper, Clemson University (2006).

11. Chandra, P. and Fisher, M.L. "Coordination of production and distribution planning", European Journal of Operational Research, 72(3), pp. 503-517 (1994).

12. Chen, Z.L. and Vairaktarakis, G.L. "Integrated scheduling of production and distribution operations", Management Science, 51(4), pp. 614-628 (2005).

13. Pundoor, G. and Chen, Z.L. "Scheduling a productiondistribution system to optimize the tradeoff between delivery tardiness and total distribution cost", Naval Research Logistics, 52(6), pp. 571-589 (2005).

14. Chen, Z.L., Handbook of Quantitative Supply Chain Analysis: Modeling in the EBusiness Era., Kluwer Academic Publishers, Norwell, MA, USA (2004). 
15. Lee, C.Y. and Chen, Z.L. "Machine scheduling with transportation considerations", Journal of Scheduling, 4(1), pp. 3-24 (2001).

16. Zhong, W.Y., Dosa, G. and Tan, Z.Y. "On the machine scheduling problem with job delivery coordination", European Journal of Operational Research, 182(3), pp. 1057-1072 (2007).

17. Hajiaghaei Keshteli, M. and Aminnayeri, M. "Solving the integrated scheduling of production and rail transportation problem by Keshtel algorithm", 25, pp. 184203 (2014).

18. Saidi Mehrabad, M., DehnaviArani, S., Evazabadian, F. and Mahmoodian, V. "An ant colony algorithm (ACA) for solving the new integrated model of job shop scheduling and conflict-free routing of AGVs", 86, pp. 2-13 (2015).

19. Chen, H.k., Hsue, C.F. and Chang, M.S. "Production scheduling and vehicle routing with time windows for perishable food products", Computers \& Operation Research, 36(7), pp. 2311-2319 (2009).

20. Liu, Z., Zhang, Y. and Li, M. "Integrated scheduling of ready-mixed concrete production and delivery", Automation in Construction, 48, pp. 31-43 (2014).

21. Averbakh, I. and Xue, Z. "On-line supply chain scheduling problems with Preemption", European Journal of Operational Research, 181(1), pp. 500-504 (2007).

22. Zegordi, S.H., Abadi, I.N.K. and BeheshtiNia, M.A. "A novel genetic algorithm for solving production and transportation scheduling in a two-stage supply chain", Computers \& Industrial Engineering, 58(3), pp. 373381 (2007).

23. Cheng, B.Y., Joseph, Y.T. and Li, K. "Integrated scheduling of production and distribution to minimize total cost using an improved ant colony optimization method", Computers \& Industrial Engineering, 83, pp. 217-225 (2015).

24. Miller, C.E., Tucker, A.W. and Zemlin, R.A. "Integer programming formulations and traveling salesman problems", Journal of the ACM, 7, pp. 326-329 (1960).

25. Kyparisis, G.J. and Koulamas, C. "Flexible flow shop scheduling with uniform parallel machines", European Journal of Operational Research, 168(3), pp. 985-997 (2006).

26. Pinedo, M.L., Scheduling: Theory, Algorithms, and Systems, Springer Science \& Business Media (2012).

27. Garey, M.R. and Johnson, D.S., Computer and Intractability, W.H. Freeman and Company, New York (1979).

28. Kulkarni, N.K., Patekar, S., Bhoskar, T. and Kulkarni, O. "Particle swarm optimization application to mechanical engineering - a review", Material Today: Proceeding, 2(4), pp. 2631-2639 (2015).
29. Guo, Y.W., Li, W.D., Mileham, A.R. and Owen, G.W. "Application of particle swarm optimization in integrated process planning and scheduling", Robotics and Computer-Integrated Manufacturing, 25(2), pp. 28-288 (2009).

30. Kennedy, J. and Eberhart, R.C. "Particle swarm optimization", Paper Presented at the Proceedings of IEEE International Conference on Neural Networks, Australia, pp. 1942-1948 (1995).

31. Banks, A., Vincent, J. and Anyakoha, C. " A review of particle swarm optimization", Part I: Background and Development, Natural Computing, 6(4), pp. 467-484 (2007).

32. Clerc, M., Particle Swarm Optimization, ISTE Ltd, London (2006).

33. Zegordi, S.H., Itoh, K. and Enkawa, T. "A knowledgeable simulated annealing scheme for the early/tardy flow shop scheduling problem", International Journal of Production Research, 33(5), pp. 1449-1466 (1995).

34. Hicks, C.R., Fundamental Concepts in the Design of Experiments, 4th Ed., Oxford University Press, New York, (1993).

\section{Biographies}

Fateme Marandi is a $\mathrm{PhD}$ student in the Department of Industrial Engineering at Amirkabir University, Iran. She received her MSc degree from the Department of Industrial Engineering at Tarbiat Modares University in 2012. Her main areas of research include production planning and scheduling, optimization problems, and meta-heuristics.

Seyed Hesameddin Zegordi is an Associate Professor of Industrial Engineering in the School of Engineering at Tarbiat Modares University, Iran. He received his $\mathrm{PhD}$ degree from the Department of Industrial Engineering and management at Tokyo Institute of Technology, Japan, in 1994. He holds an MSc degree in Industrial Engineering and Systems from Sharif University of Technology, Iran, and a BSc degree in Industrial Engineering from Isfahan University of Technology, Iran. His main areas of teaching and research interests include production planning and scheduling, multi-objective optimization problems, meta-heuristics, quality management, and productivity. He has published several articles in international conferences and academic journals including European Journal of Operational Research, International Journal of Production Research, Journal of Operational Research Society of Japan, Computers \& Industrial Engineering, Amirkabir Journal of Science and Engineering, and Scientia Iranica International Journal of Science and Technology. 